\title{
Une approche instrumentale de l'éducation aux débats socio-scientifiques en ligne
}

\author{
An instrumental approach to education \\ for online socio-scientific debates
}

\section{Un enfoque instrumental de la educación para el debate socio-científico en línea}

Jean-Marc Meunier, maitre de conférences Laboratoire Paragraphe, université Paris 8, France imeunier@univ-paris8

RÉSUMÉ

De notre point de vue, l'enseignement en ligne est par essence une activité de collaboration médiatisée, plus encore lorsqu'il s'appuie sur une pédagogie active et met en scène les interactions entre étudiants. Les outils dont disposent les enseignants sont multiples, parfois complexes, et s'organisent en système qu'il faut pouvoir analyser pour comprendre une activité telle qu'un débat en ligne. Dans cet article, nous proposons l'analyse d'une telle situation dans le cadre de l'approche instrumentale (Lonchamp, 2012; Rabardel et Beguin, 2005; Rabardel, 1995) afin de montrer comment la notion de compétence, telle qu'elle est proposée dans ce cadre, peut permettre de penser l'alignement entre les activités pédagogiques, les compétences visées et l'évaluation de ces dernières.

Mots-clés : apprentissage collaboratif en ligne, approche instrumentale, schème, formation à la pensée scientifique

\section{ABSTRACT}

From our point of view, online education is inherently a mediated collaborative activity, even more so when it is based on active pedagogy and involves student interaction. The tools available to teachers are multiple, sometimes complex, and are organized into a system that must be analyzed in order to understand an activity such as an online debate. In this article, we propose the analysis of such a situation in the framework of the instrumental approach (Lonchamp, 2012; Rabardel \& Beguin, 2005; Rabardel, 1995) in order to show how the notion of competence, as proposed in this framework, can allow us to think about the alignment between pedagogical activities, the targeted competencies and the evaluation of them. 
Keywords: online collaborative learning, instrumental approach, scheme, scientific thinking education

RESUMEN

Desde nuestro punto de vista, la educación en línea es inherentemente una actividad de colaboración mediada, más aún cuando se basa en una pedagogía activa e involucra la interacción de los estudiantes. Los instrumentos de que disponen los profesores son múltiples, a veces complejos, y están organizados en un sistema que debe poder analizarse para comprender una actividad como un debate en línea. En el presente artículo proponemos el análisis de esa situación en el marco del enfoque instrumental (Lonchamp, 2012; Rabardel \& Beguin, 2005; Rabardel, 1995) a fin de mostrar cómo la noción de competencia, tal como se propone en este marco, puede permitir pensar en la alineación entre las actividades pedagógicas, las competencias específicas y la evaluación de estas últimas.

Palabras clave: aprendizaje colaborativo en línea, enfoques instrumentales, esquema, formación en pensamiento científico

\section{L'enseignement à distance : une situation de collaboration en ligne}

Le vocable d' " enseignement à distance » laisse trompeusement croire que la principale caractéristique de cette forme d'enseignement est la distance géographique entre l'enseignant et l'apprenant. La distance peut également être temporelle si on considère la dimension asynchrone de la plupart des interactions qui la caractérisent. Ces points de vue conduisent dans le langage courant à opposer l'enseignement à distance au présentiel. Pourtant, même si la forme diffère, l'enseignement à distance nécessite également une présence et des formes de régulation. II n'y a pas moins de présence dans une situation d'enseignement à distance que dans une situation d'enseignement présentiel. L'apprenant n'est pas non plus mis dans une situation passive (Jézégou, 2012). L'environnement pédagogique qu'on lui propose, typiquement la plateforme d'enseignement, n'est pas clos. II est ouvert sur d'autres sources documentaires, sur d'autres individus et éventuellement d'autres lieux d'interaction comme les réseaux sociaux. Pour évoluer dans un tel environnement, l'apprenant doit cependant développer des formes d'autorégulation spécifiques (Jézégou, 2010). La généralisation de l'usage d'Internet et le développement des pédagogies actives font finalement de cette forme d'enseignement une situation par essence collaborative. Elle a cependant la particularité d'être médiée et médiatisée par la production et l'échange de documents numériques et orientée vers l'acquisition de connaissances et de compétences, propriétés qui finalement caractérisent tout cadre éducatif (Béguin et Rabardel, 2000).

De notre point de vue, apprendre à distance consiste à naviguer dans un écosystème documentaire dynamique, ce que Szoniecky a comparé à la culture d'un jardin de connaissances (Szoniecky et Meunier, 2020; Szoniecky, 2018) dans lequel les apprenants vont produire, transformer ou hybrider des documents. Les documents ont une double fonction : formaliser des questions, des idées ou des connaissances, et susciter un feedback de l'enseignant ou de la part des autres étudiants, pour obtenir un complément d'information, en tester la cohérence, défendre son point de vue ou faire la preuve de ses acquisitions. Cette double fonction, formaliser et agir sur l'autre, confère aux documents une dimension instrumentale, tant pour l'enseignant que pour l'étudiant. 
Pour un enseignant, les documents sont des instruments qui fournissent à l'élève, par leur mise en scène, un moyen d'acquisition des connaissances et des compétences visées dans le cours en prescrivant des activités sur ceux-ci. Le même point de vue peut être adopté pour l'étudiant dans la mesure où le devoir, les interventions sur les forums ou les traces d'activités qu'il donne à voir sont autant d'instruments permettant de faire comprendre à l'enseignant et aux autres étudiants ce qui a été acquis, ce qui pose problème ou doit être discuté. Cependant, ce qui donne sa valeur instrumentale à un document ou à une trace d'activité, ce n'est pas l'intention du producteur, mais la capacité du récepteur à le traiter comme tel. II nous faut donc pouvoir appréhender les schèmes que chacun des protagonistes peut ou non mettre à l'œuvre pour cela.

\section{L'approche instrumentale}

L'approche instrumentale à laquelle nous nous référons est issue des travaux de Rabardel (1995) visant à rendre compte des activités médiées par des instruments. Les instruments sont des entités mixtes composés à la fois d'un outil (artefact) qui correspond à la partie technique et d'un sujet qui l'utilise grâce à des schèmes d'action. L'outil acquiert sa dimension instrumentale au cours d'un processus appelé genèse instrumentale. Cette approche permet de décrire la situation d'enseignement à distance au niveau des propriétés globales de la situation (niveau macro). Nous allons les examiner dans une première partie de cet article en présentant notre cadre conceptuel. L'approche instrumentale permet aussi d'analyser la façon dont les utilisateurs réels s'approprient l'outil pour atteindre un but dans un contexte particulier (niveau micro). Nous l'aborderons dans la seconde partie de cet article lorsque nous présenterons l'analyse de notre dispositif pédagogique.

\section{LE CARRÉ DIDACTIQUE}

Au niveau macro, le rôle des instruments est de permettre la mise en forme et la diffusion des notions, des contenus, et de présenter les activités prescrites (médiatisation), ce que Rabardel nomme une instrumentalisation, c'est-à-dire une mise en forme pour servir d'instrument. Dans une situation d'apprentissage, ils servent ainsi d'intermédiaire entre le savoir et l'apprenant. Pour l'apprenant, ils constituent un intermédiaire entre lui et le savoir. Cela correspond à un processus d'instrumentation qui a pour objet la construction et l'adaptation des schèmes d'utilisation des instruments par l'apprenant (médiation). Si on considère que le langage, les formalismes, les activités pédagogiques et les documents utilisés par l'enseignant sont autant d'instruments, il n'y a guère d'activités pédagogiques qui ne soient instrumentées. S'appuyant sur cette approche, Rézeau (2002) a ajouté au triangle didactique classique (apprenant, savoir, enseignant) un quatrième pôle constitué par les instruments qui peuvent être des supports, des activités, des tâches ou tout type de matériels, mais aussi des instruments psychologiques, tels que le langage ou les concepts. Plus récemment, Lonchamp (2012) a également proposé un modèle quadripolaire analogue dans son analyse des systèmes d'apprentissage collaboratif par ordinateur.

\section{LE CYCLE INSTRUMENTAL}

Nous avons utilisé ce carré didactique pour formaliser une situation de travail collaboratif en ligne. La modélisation que nous en proposons dans la figure 1 est bien sûr une simplification. Elle ne considère que les échanges de documents. Les instruments nécessaires à la production et à la diffusion des documents sont laissés de côté. Dans cette figure, chaque losange représente un document ou un ensemble de documents considérés comme instrument. Tout document a un auteur représenté par le cartouche de la pointe inférieure du losange. Le ou les destinataires sont symbolisés par la pointe supérieure du losange. L'axe horizontal représente la relation entre la conceptualisation du sujet (en gros, leur savoir ou la thèse défendue) et l'objet, c'est-à-dire le ou les documents en tant qu'artefact. 


\section{Figure 1}

Transposition du carré didactique de Rézeau (2002) à l'analyse du travail collaboratif dans le cadre de l'enseignement à distance

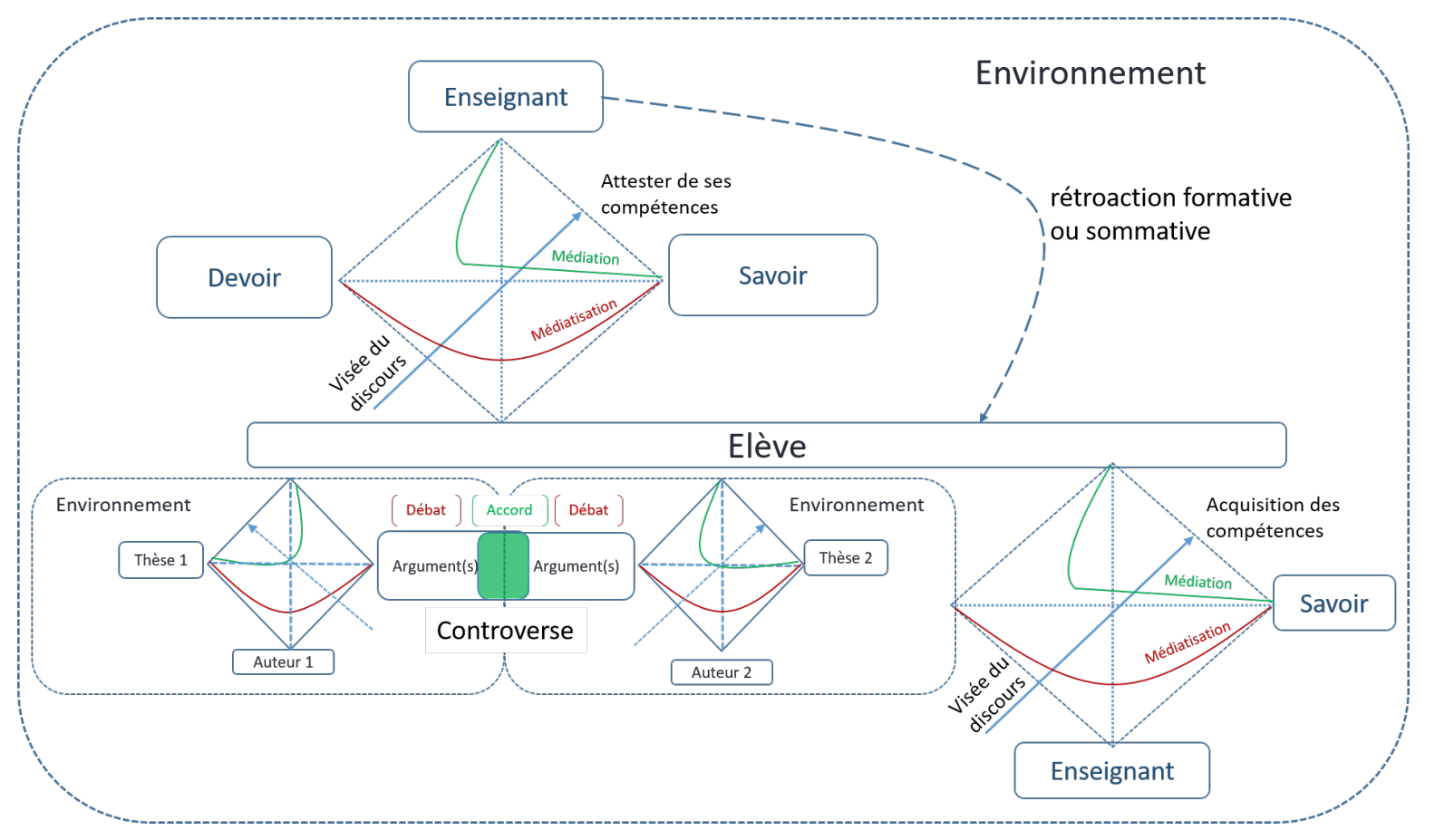

Le modèle s'applique de manière récursive. Ainsi lorsqu'un enseignant prépare son cours, il rassemble ou produit un ensemble de documents. Pour susciter les débats en ligne, nous avons par exemple fourni à nos étudiants une base de données bibliographiques (Meunier et Zibetti, 2019). Les documents choisis notamment pour leur divergence sont instrumentalisés pour amener les étudiants à identifier les controverses, les arguments et les termes du débat. Chacun des documents a un auteur qui défend un point de vue ou, si on préfère, une thèse et le donne à voir au destinataire sous une forme particulière (organisation des arguments, documents mis à disposition, etc.). Parce que l'enseignant prescrit la lecture de ces documents aux apprenants, ceux-ci deviennent les destinataires de ces documents et peuvent ainsi se les approprier. Ils vont pouvoir en surligner certains passages, les annoter et confronter leur point de vue avec les autres étudiants. Ce faisant, il réalise ce que Szoniecky (2018) appelle une métamorphose, c'est-à-dire la création d'un nouveau document par modification d'une de ces instances. Pour faire retour à l'enseignant, seul ou en groupe, les apprenants vont alors produire un nouveau document, généralement en agençant les idées trouvées dans les précédents (sans oublier de les citer). Szoniecky parle alors d'hybridation et conçoit cette dernière comme une métamorphose enrichie. Enfin, le travail de l'enseignant consiste à opérer une nouvelle métamorphose du document en commentant la copie et en fournissant ainsi un feedback à l'étudiant. 


\section{La pluralité des formes de médiation}

Une caractéristique importante des activités instrumentées est la pluralité des formes de médiation. Chaque utilisation d'un instrument s'inscrit dans plusieurs formes de médiation, même si certaines formes peuvent dominer en fonction des situations et des sujets (Cerratto Pargman et al., 2018). On distingue quatre formes de médiation dans les situations d'apprentissage collaboratif (Lonchamp, 2012; Rabardel et Bourmaud, 2003). Les deux premières concernent la relation entre le sujet (l'élève ou l'enseignant) et l'objet (le savoir). La médiation épistémique donne à comprendre l'objet à travers l'instrument. C'est typiquement appréhender un concept ou une théorie (objet) à l'aide de documents (instruments). La position d'objet n'est pas intrinsèque, mais dépend de l'activité et de l'instrument utilisé dans celle-ci. Ainsi, le document peut aussi être placé en position d'objet (ce qu'on cherche à connaitre) lorsqu'on souhaite mettre en évidence les passages importants avec des annotations (instrument). Le second type de médiation est pragmatique et est orienté vers la transformation de l'objet et l'obtention d'un résultat. Par exemple, l'annotation conduit à la transformation du document avec pour résultat la création d'un résumé ou l'identification des lignes de force d'un texte, si on ne considère que les passages annotés.

À ces deux formes de médiation concernant l'axe sujet-objet, Lonchamp (2012) ajoute une médiation concernant l'axe interpersonnel. Dans la figure 1, c'est l'axe qui relie l'auteur (ou locuteur) et le destinataire (ou interlocuteur). Comme pour l'axe sujet-objet, la médiation interpersonnelle est déclinée entre une médiation épistémique, visant à connaitre l'autre, et pragmatique, c'est-à-dire visant à agir sur eux. Le post sur un forum peut servir ces deux types de médiation en sollicitant par exemple un enseignant à donner un complément d'information.

La quatrième forme est la médiation réflexive (ou médiation heuristique) qui s'inscrit dans la relation du sujet à lui-même à travers l'instrument (Samurçay et Rabardel, 2004). C'est typiquement ce qu'on fait lorsqu'on cherche à tester sa représentation à l'aide d'un document, en postant une question sur un forum ou lorsqu'on insère une marque dans une annotation pour faire le lien avec une autre idée ou se rappeler quelque chose.

\section{Tâches, activités et schèmes instrumentaux}

Un des principaux intérêts de la notion d'instrument pour l'analyse des situations d'enseignement collaboratif réside dans la prise en compte d'un double mouvement : l'utilisation d'un instrument conduit à la fois à la découverte des propriétés de celui-ci, mais aussi à l'adaptation des schèmes. Comme dans n'importe quelle tâche médiée par un instrument, le sujet doit gérer simultanément deux tâches, l'une principale et l'autre secondaire. Dans notre exemple, la première concerne le traitement du document luimême, sa structure, le titre, le résumé, les niveaux de titres ou la bibliographie et autres éléments de paratextes qui en facilitent l'exploitation. La tâche secondaire est relative à l'utilisation. Elle dépend du dispositif de consultation du document. Ce peut-être une version papier, un lecteur PDF ou un navigateur. Ces deux tâches mettent en œuvre des schèmes différents. Le premier niveau renvoie aux habiletés pour la lecture et l'analyse de texte, le second à l'utilisation d'un dispositif technique.

Dans les deux cas, l'activité est organisée par un certain nombre d'invariants. Ce sont les éléments constitutifs des schèmes. Ainsi, pour faire l'analyse d'un document, nous recommandons généralement à nos étudiants de lire d'abord le résumé, s'il y en a un, puis de prendre connaissance des titres et intertitres pour se faire une idée globale, avec éventuellement une première lecture rapide avant de faire une lecture approfondie, le surligneur à la main, qu'il soit physique ou numérique. II s'agit du schème général de l'analyse d'un texte académique. L'activité peut également être organisée en une série de tâches dans lesquelles nous retrouverons à nouveau la distinction entre la tâche proprement dite et l'utilisation de l'outil. 
Si nous considérons par exemple l'annotation d'un document, une telle activité nécessite la coordination d'au moins deux schèmes, l'un pour traiter le document lui-même : que surligner? dans quelle limite (motsclés, phrase ou paragraphe)?, typologie des annotations? etc.; l'autre pour utiliser le dispositif d'annotation. S'agissant d'un feutre, cela ne semble pas poser de question tant les schèmes semblent bien intégrés, mais dans le cas des documents informatiques, il en va autrement. Avec un lecteur PDF ou un traitement de texte, il est possible d'annoter et de commenter, mais contrairement à l'annotation manuelle, il faut sélectionner préalablement ce qui doit être surligné ou annoté. Selon les dispositifs, l'annotation peut rester apparente en parallèle du texte ou être masquée, associée au surlignement ou indépendante. En ce qui concerne les documents en ligne (page Web ou PDF), il faut recourir à un outil d'annotation qui nécessite un apprentissage spécifique, plus encore si on veut l'utiliser pour un travail collaboratif.

On peut distinguer trois types de schèmes (Rabardel, 1995). Les premiers sont les schèmes d'utilisation. Ils sont orientés vers l'utilisation de l'outil lui-même, c'est-à-dire le dispositif d'annotation dans notre exemple précédent. Viennent ensuite les schèmes d'action à l'aide de l'instrument. Ils concernent la tâche principale. Dans notre exemple, c'est la compréhension du document par identification des passages importants à l'aide de l'outil d'annotation. La dernière catégorie concerne plus spécifiquement les activités collaboratives qui nous intéressent. Ce sont les schèmes d'activités collectives. C'est grâce à eux qu'un groupe d'individus coordonne et régule les interactions et les productions pour parvenir au but commun. La gestion des réunions, par exemple, nécessite de tels schèmes. Ils permettent de trouver un créneau commun, de considérer un ordre du jour négocié ou imposé et de gérer les tours de paroles, éventuellement la proposition de synthèse intermédiaire puis la formulation d'une conclusion. Dans le cas de la production d'un document collectif, ces schèmes d'activité collective vont concerner l'utilisation d'un instrument commun autant que la gestion de l'activité elle-même. II faut ainsi se mettre d'accord (i) sur le choix de l'outil (édition en ligne, dossier partagé, gestionnaire de version, etc.) et s'assurer que tout le monde a les schèmes d'utilisation ou peut les acquérir, (ii) le mode de gestion des différentes versions (centralisation des propositions, correction directe dans le document ou commentaire et annotations) et (iii) le processus d'arbitrage entre les différentes propositions (répartition des responsabilités sur les différentes parties, qui fait la synthèse ou décide en cas de litige).

\section{Le projet PEPE}

Le cadre théorique que nous venons de présenter permet d'analyser un dispositif de formation à un niveau macro, mais aussi à un niveau micro. C'est ce dont nous allons discuter en présentant un dispositif de formation à la recherche élaboré dans le cadre d'un parcours pluriannuel en licence de psychologie. Notre approche pédagogique consiste à penser l'initiation à la recherche de manière progressive en distinguant trois étapes: (i) le développement des compétences à la recherche documentaire critique, (ii) les compétences permettant de comprendre et de prendre part à une controverse socio-scientifique et enfin (iii) les compétences permettant de mener une recherche proprement dite, notamment la problématisation de la controverse et son opérationnalisation. Nous avons fait coïncider ces étapes avec les trois années de licence. Le cours est dispensé entièrement à distance et les communications se font de façon asynchrone à l'exception des travaux dirigés où nous utilisons la visioconférence.

Ce dispositif de formation a été publié sur une plateforme Omeka-s (http://pepe.univ-paris8.fr), dans le cadre d'un projet baptisé PEPE (Penser, s'Etonner, Problématiser, Evaluer) et financé par la communauté d'universités et établissements (COMUE) Université Paris Lumière (Meunier et Zibetti, 2019). Cette plateforme permet à la fois de gérer les ressources et d'éditorialiser les contenus. Nous avons utilisé une base de données bibliographiques et un outil d'annotation, Hypothesis (https://web.hypothes.is/), pour que les étudiants de première année réalisent une analyse argumentative sur le document de leur choix et en fassent un compte-rendu de lecture critique. En seconde année, c'est le débat, à l'aide du même outil 
d'annotation et l'analyse croisée de documents, qui permet de travailler sur les controverses. Nous nous appuyons pour cela sur les compétences à l'analyse critique construites en première année. Ce travail est finalisé par la production d'un rapport d'étonnement, c'est-à-dire l'identification et la formalisation des termes d'une controverse (ce sont les arguments en débat dans la figure 1). L'étonnement attendu n'est pas l'effet de surprise, mais l'étonnement épistémique indispensable à une possible problématisation (Fabre, 2014). Cet étonnement permettra en troisième année de travailler sur les conditions de résolution de la controverse, c'est-à-dire la problématisation et l'opérationnalisation de celle-ci, et sera concrétisé par une petite recherche dans le cadre d'un projet tuteuré.

\section{ORGANISATION DES DÉBATS EN LIGNE}

Jusqu'à maintenant, les étudiants en seconde année devaient travailler individuellement l'analyse des controverses. À partir d'octobre 2020, ce travail est réalisé en petit groupe. C'est l'analyse de ce nouveau dispositif que nous proposons maintenant. Ce changement intervient après la formation d'une première promotion de Licence première année (L1) à l'utilisation de l'outil d'annotation Hypothesis sur lequel le dispositif de deuxième année (L2) s'appuie techniquement. Le scénario pédagogique est le suivant : après une formation aux débats scientifiques qui fait écho à la formation épistémologique et méthodologique qu'ils ont par ailleurs, les étudiants sont invités à choisir un thème dans une liste de débats socioscientifiques relatifs à l'impact du numérique sur les processus sociocognitifs. Les étudiants peuvent également en proposer un. Un thème se présente sous la forme d'une affirmation, dont voici deux exemples :

- L'usage des écrans a un impact négatif sur le développement cognitif des enfants.

- L'addiction au jeu est une forme d'addiction à part entière.

Les étudiants travaillent en groupe de six, un même thème pouvant être traité par plusieurs groupes. Les étudiants doivent se répartir en deux camps, les défenseurs et les accusateurs de la thèse, puis opérer une sélection d'un document chacun qui servira de base au débat. La tâche de chacun consiste à convaincre l'autre camp qu'il a raison. Les échanges autour de ces documents se feront de façon asynchrone dans un groupe privé sous Hypothesis. Le travail final attendu est un rapport d'étonnement exposant l'état de la controverse, les arguments pour (thèse) et contre (antithèse), ainsi qu'une synthèse. Les activités à mettre en œuvre pour réaliser le travail attendu sont listées dans le tableau suivant sous la forme d'acquis d'apprentissage visés (AAV), c'est-à-dire de comportements observables attendus à l'issue de la formation (Warnier et al., 2010).

\section{Tableau 1}

Les activités de l'étudiant lors d'un débat en ligne

\begin{tabular}{|c|c|c|}
\hline $\begin{array}{c}\text { Utilisation de dispositifs } \\
\text { techniques }\end{array}$ & Activités individuelles & Activités collectives \\
\hline $\begin{array}{l}\text { Utiliser un calendrier ou un } \\
\text { gestionnaire de tâches }\end{array}$ & \multicolumn{2}{|c|}{$\begin{array}{l}\text { Organiser un débat } \\
\text { - Définir l'organisation temporelle } \\
\text { - Règles d'échange }\end{array}$} \\
\hline Utiliser une base de données & \multirow{2}{*}{$\begin{array}{l}\text { Sélectionner un document } \\
\text { - Formuler une requête } \\
\text { - Évaluer les résultats }\end{array}$} & \multirow{2}{*}{$\begin{array}{l}\text { Négocier le fond documentaire } \\
\text { commun }\end{array}$} \\
\hline Faire une recherche sur Internet & & \\
\hline
\end{tabular}




\begin{tabular}{|l|l|l|}
\hline \multicolumn{1}{|c|}{$\begin{array}{c}\text { Utilisation de dispositifs } \\
\text { techniques }\end{array}$} & \multicolumn{1}{c|}{ Activités individuelles } \\
\hline $\begin{array}{l}\text { Utiliser Hypothesis } \\
\text { - Créer un groupe } \\
\text { - Associer des documents } \\
\text { - Annoter } \\
\text { - Commenter des annotations }\end{array}$ & $\begin{array}{l}\text { Analyser un texte socio- } \\
\text { scientifique } \\
\text { - Repérer les arguments } \\
\text { - Évaluer les arguments }\end{array}$ & $\begin{array}{l}\text { Prendre part à un débat socio- } \\
\text { scientifique } \\
\text { - Défendre son point de vue } \\
\text { - Réfuter le point de vue opposé } \\
\text { - Élaborer une synthèse }\end{array}$ \\
\hline $\begin{array}{l}\text { Utiliser un document partagé } \\
\text { - Créer le document }\end{array}$ & $\begin{array}{l}\text { Organiser un travail de rédaction } \\
\text { - Partager le document }\end{array}$ & $\begin{array}{l}\text { - Structurer le document } \\
\text { - Répartir des tâches de rédaction } \\
\text { - Définir des règles de validation }\end{array}$ \\
\hline
\end{tabular}

Bien qu'utiles pour analyser à grands traits une formation, ces AAV ne nous disent rien des processus à l'œuvre pour obtenir ces résultats et donc, finalement, rien des compétences sous-jacentes ni ce qu'il convient de faire pour remédier aux difficultés éventuelles. Il est donc important d'ouvrir la boite noire.

\section{L’ANALYSE DES COMPÉTENCES}

Les compétences, dans l'approche instrumentale, sont envisagées comme des structures d'organisation de l'activité et décrites par des schèmes dont les composants (invariants opératoires, inférences, règles d'action, anticipations) permettent de préciser la nature des observables associés à leur mise en œuvre, c'est-à-dire la performance (Coulet, 2011; Loisy et Coulet, 2018), donnant ainsi corps à la notion d'acquis visés d'apprentissage. La figure 2 présente l'alignement entre les composants des schèmes et les prises d'information par l'enseignant à travers les outils pédagogiques qu'il mobilise.

\section{Figure 2}

Analyse de l'activité instrumentée de l'élève et de la situation pédagogique, d'après le modèle de Coulet (2011)

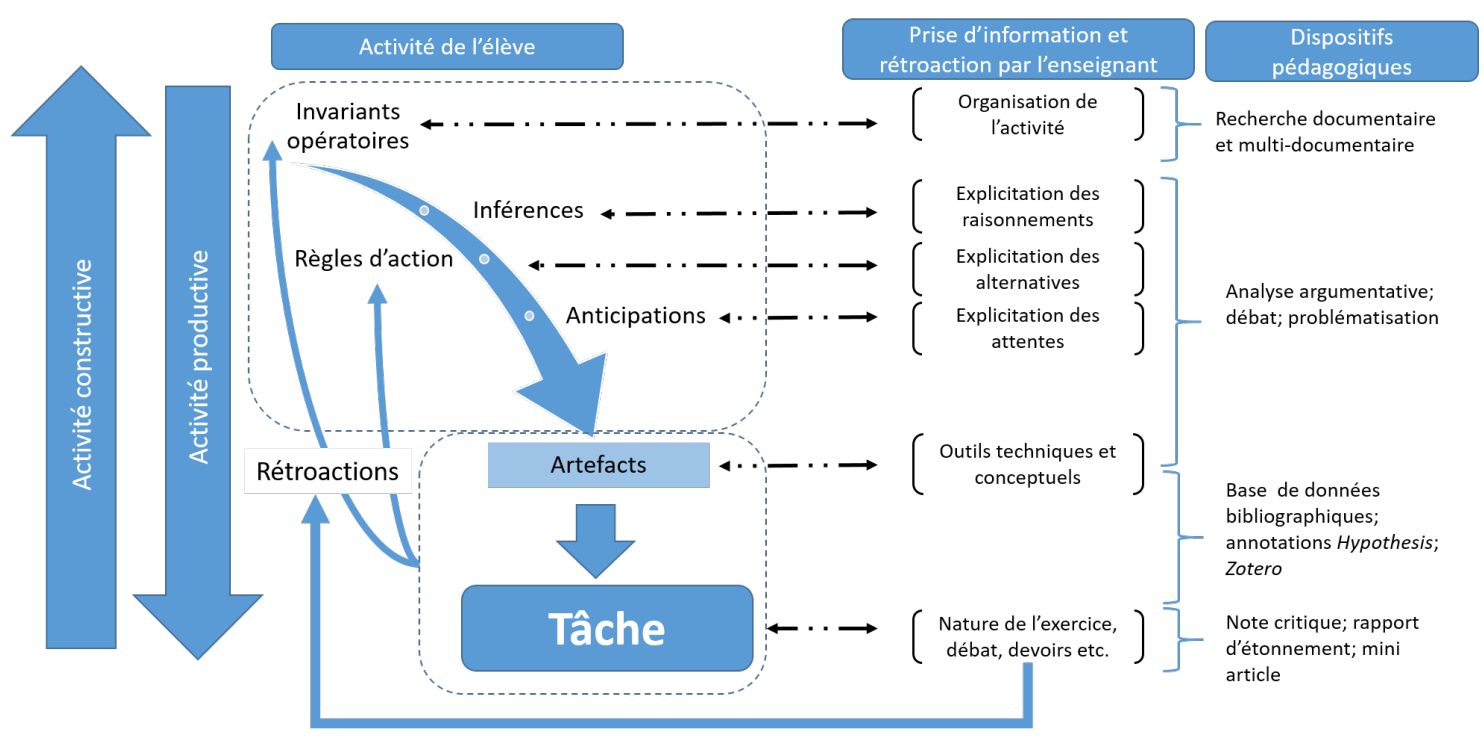

Nous faisons l'hypothèse que les individus mettent en œuvre un ou plusieurs schèmes pour réaliser chacune de ces activités. Dans le tableau 1, certaines activités concernent l'utilisation des outils et renvoient donc à des schèmes d'utilisation. Les autres concernent des activités individuelles ou collectives. Certaines activités, notamment les activités organisationnelles, sont à cheval entre les activités 
individuelles et collectives. Les schèmes ont parfois été construits antérieurement et doivent être ajustés. Ainsi, la participation à un débat à l'aide d'Hypothesis nécessite de savoir utiliser celui-ci et d'analyser de manière critique des documents, ce que les étudiants ont appris en L1. En L2, nous ne faisons donc qu'ajouter les dimensions sociocognitive et multidocumentaire à ce travail de compréhension des controverses, ce qui va nécessiter l'adaptation des schèmes antérieurement acquis. Les schèmes ne sont cependant pas directement observables. On ne peut y accéder qu'à travers le résultat de l'activité. Les $\mathrm{AAV}$ n'en constituent qu'une description grossière.

Pour bien comprendre le processus d'élaboration et surtout penser l'alignement pédagogique entre les compétences visées, les activités proposées et l'évaluation, il nous faut une description plus fine que les $\mathrm{AAV}$ et surtout un moyen de suivre le développement des compétences. Ce moyen n'est pas donné par le devoir final qui est une reconstruction, qui plus est collective, mais par les annotations sous Hypothesis qui constituent des traces du processus de débat. Elles sont, en effet, individuelles et datées. II est alors possible d'analyser finement les échanges argumentatifs (Checchi et Pallares, 2020; Pallarès, 2019). De telles analyses permettent ainsi à l'enseignant une prise d'information sur chacune des composantes des schèmes et d'agir sur celle-ci et la situation pédagogique (voir figure 2).

\section{Discussion}

Le cadre théorique dans lequel nous situons notre dispositif pédagogique offre un puissant outil conceptuel pour le penser à la fois au niveau macro et au niveau micro. De notre point de vue, les dispositifs d'enseignement à distance sont des systèmes d'apprentissage collaboratif en ligne. Ces systèmes peuvent être décrits comme des systèmes instrumentaux dans lesquels les documents ont un statut particulier, à la fois produits et instruments utilisés tant par l'enseignant que par l'étudiant pour gérer les échanges et acquérir des connaissances et des compétences. Les étudiants peuvent cependant s'écarter de l'intention pédagogique souhaitée par l'enseignant dans sa prescription de l'usage du dispositif (voir, par exemple, Demory et Girel, 2019). Une première façon de gérer cet écart est de renforcer le tutorat et éventuellement de lui adjoindre des moyens informatiques : Leaning analytics, agents intelligents etc. (Magnisalis et al., 2011). Nous nous situons plutôt du côté de ce que Lonchamp (2012) nomme "les systèmes instrumentalisables par les apprenants ", c'est-à-dire laissant aux apprenants la liberté de s'emparer à leur façon du dispositif. Nous voyons à une telle approche plusieurs avantages. Elle est d'abord techniquement plus simple et compatible avec les cohortes importantes qui sont les nôtres. En effet, en L2, nous avons plus de 100 étudiants et un seul enseignant pour s'en occuper. Par ailleurs, le projet s'inscrit dans une démarche d'autonomisation des étudiants. Notre intention est de les engager dans une démarche d'instrumentalisation des documents et des codes du débat scientifique. De ce point de vue, il est beaucoup plus opérationnel de chercher à appréhender les schémas d'activités mis en œuvre par les étudiants dans leur utilisation collective du dispositif. En effet, l'évaluation de celui-ci ne peut avoir de sens, dans le cadre d'une approche instrumentale, que dans l'évaluation de la signification pour un utilisateur particulier dans une tâche bien définie (White, 2008). Parallèlement, l'évaluation de l'apprenant ne prend sens qu'au regard des schèmes qu'il a mis en œuvre pour appréhender l'activité et sa capacité à les faire évoluer pour attendre le but prescrit. Ce qui importe, c'est sa trajectoire cognitive. Les annotations des apprenants constituent de ce point de vue une fenêtre ouverte sur ces processus. Elles vont permettre d'étudier la mise en œuvre des différents types de schèmes et la genèse instrumentale chez l'apprenant. L'enjeu est maintenant l'élaboration d'une méthodologie d'analyse qui constitue un projet de recherche en soi, tant pour mettre en évidence les schèmes postulés que pour comprendre la dynamique de leur évolution. Le point de vue que nous avons sur le document implique par ailleurs que les processus d'instrumentation et d'instrumentalisation sont autant du côté de l'enseignant que de l'étudiant. Or, « les deux processus contribuent conjointement, et souvent de manière dialectique, à la construction et à l'évolution de l'instrument » (Béguin et Rabardel, 2000, p. 181). Cela constitue le 
dernier avantage d'adopter le point de vue des systèmes instrumentalisables par les apprenants. En situant le dispositif dans cette dialectique, on met en place les conditions de son évolution, ce qui est une autre façon de dire qu'on souhaite une démarche centrée sur l'apprenant et de l'inscrire dans une démarche qualité qui dépasse la simple évaluation de sa perception par les étudiants.

\section{Liste de références}

Béguin, P. et Rabardel, P. (2000). Designing for instrument-mediated activity. Scandinavian Journal of Information Systems, 12(1), 1.

Cerratto Pargman, T., Nouri, J. et Milrad, M. (2018). Taking an instrumental genesis lens: New insights into collaborative mobile learning. British Journal of Educational Technology, 49(2), 219-234.

Checchi, K. D. et Pallares, G. (2020). «Dans tous les cas, il y a un bon avis mais... contre un autre »: Mettre en lien la qualité de l'argumentation et l'épistémologie personnelle de l'élève. Educational Journal of the University of Patras UNESCO Chair, 7(1). https://pasithee.library.upatras.gr/ejupUNESCOchair/article/view/3162

Coulet, J.-C. (2011). La notion de compétence : Un modèle pour décrire, évaluer et développer les compétences. Le travail humain, 74(1), 1-30. https://doi.org/10.3917/th.741.0001

Demory, M. et Girel, S. (2019). Accessibilités, usages et appropriations des technologies numériques : Médiations et médiatisations, 2, 187-198. https://revue-mediations.teluq.ca/index.php/Distances/article/view/79

Fabre, M. (2014). De l'étonnement au problème. Education Permanente, 4(200), 97-105.

Jézégou, A. (2010). Se former à distance : Regard sur les stratégies d'autorégulation environnementale d'étudiants adultes. Savoirs, 24(3), 79-99.

Jézégou, A. (2012). La présence en e-learning: Modèle théorique et perspectives pour la recherche. International Journal of E-Learning \& Distance Education, 26(1). http://www.ijede.ca/index.php/jde/article/view/777

Loisy, C. et Coulet, J.-C. (2018). Compétences et approche-programme : Outiller le développement d'activités responsables (vol. 2). ISTE Editions.

Lonchamp, J. (2012). An instrumental perspective on CSCL systems. International Journal of Computer-Supported Collaborative Learning, 7(2), 211-237.

Magnisalis, I., Demetriadis, S. et Karakostas, A. (2011). Adaptive and Intelligent Systems for Collaborative Learning Support: A Review of the Field. IEEE Transactions on Learning Technologies, 4(1), 5-20. https://doi.org/10.1109/TLT.2011.2

Meunier, J.-M. et Zibetti, E. (2019, janvier 17). Penser, s'Etonner, Problématiser et Evaluer (PEPE) : Un parcours pluriannuel d'initiation à la recherche en licence à distance. Education 4.1! Distances, médiations des savoirs et des formations. https://hal-univ-paris8.archives-ouvertes.fr/hal-02018386/document

Pallarès, G. (2019). Développer les compétences argumentatives de lycéens par des débats numériques sur des questions socio-scientifiques. Vers une didactique de l'argumentation et de l'esprit critique [thèse de doctorat, Université de Montpellier]. https://tel.archives-ouvertes.fr/tel-02442726

Rabardel, P. et Beguin, P. (2005). Instrument mediated activity: From subject development to anthropocentric design. Theoretical Issues in Ergonomics Science, 6(5), 429-461.

Rabardel, P. (1995). Les hommes et les technologies; approche cognitive des instruments contemporains. Armand Colin. https://hal.archives-ouvertes.fr/hal-01017462

Rabardel, P. et Bourmaud, G. (2003). From computer to instrument system: A developmental perspective. Interacting with computers, 15(5), 665-691.

Rézeau, J. (2002). Médiation, médiatisation et instruments d'enseignement: Du triangle au "carré pédagogique ». ASp, 35-36, 183-200. https://doi.org/10.4000/asp.1656

Samurçay, R. et Rabardel, P. (2004). Modèles pour l'analyse de l'activité et des compétences, propositions. Dans R. Samurçay et P. Pastré (dir.), Recherches en didactique professionnelle (p. 163-180). Octarès.

Szoniecky, S. (2018). Écosystème de connaissances: Méthode de modélisation et d'analyse de l'information et de la communication (vol. 5). ISTE Editions. 
Szoniecky, S. et Meunier, J.-M. (2020). Le jardin comme écosystème de connaissances. Intermédialités. Histoire et théorie des arts, des lettres et des techniques, Automne 2020(36).

Warnier, P., Warnier, L., Parmentier, P., Leloup, G. et Petrolito, S. (2010). Et si on commençait par les résultats? Elaboration d'une démarche de définition des acquis d'apprentissage d'un programme de formation universitaire. $26^{\mathrm{e}}$ Congrès international de l'AIPU. 17-21 mai 2010, Rabat, Maroc.

https://dial.uclouvain.be/pr/boreal/object/boreal:166153/datastream/PDF_01/view

White, T. (2008). Debugging an Artifact, Instrumenting a Bug: Dialectics of Instrumentation and Design in Technology-Rich Learning Environments. International Journal of Computers for Mathematical Learning, 13(1), 1-26. https://doi.org/10.1007/s10758-007-9119-x 